\title{
A Case of Severe, Persistent Amnesia Following an Episode of Prolonged Status Epilepticus
}

\author{
Avidesh Panday ${ }^{\mathrm{a}}$, Werner J. Becker ${ }^{\mathrm{a}, \mathrm{c}}$, Jayesh Modi ${ }^{\mathrm{b}}$
}

\begin{abstract}
The role of the hippocampus in memory loss has been well established. We present a case of persistent and profound amnesia in a patient with a prolonged episode of status epilepticus complicated by a history of alcohol abuse. Serial brain MRI's showed concomitant evolution of profound hippocampal damage.
\end{abstract}

Keywords: Hippocampal sclerosis; Memory; Status epilepticus; MRI; Epilepsy monitoring

\section{Introduction}

We present a case of persistent and profound amnesia in a patient with a prolonged episode of status epilepticus complicated by a history of alcohol abuse. Serial brain MRI's showed concomitant evolution of profound hippocampal damage.

\section{Case Report}

A 48-year-old Caucasian male was admitted to hospital with an altered level of consciousness after experiencing multiple recurrent generalized tonic clonic seizures without recovery between episodes for approximately 45 mins before presentation to hospital. Despite initiation of intravenous phenytoin

\footnotetext{
Manuscript accepted for publication March 21, 2013

${ }^{a}$ Department of Clinical Neurosciences, University of Calgary and Alberta Health Services, Calgary, Alberta, Canada

${ }^{\mathrm{b}}$ Department of Radiology, University of Calgary and Alberta Health Services, Calgary, Alberta, Canada

${ }^{\mathrm{c}}$ Corresponding author: Werner J. Becker, Division of Neurology, Foothills Hospital, 1403 29th St NW, Calgary, AB, T2N 2T9, Canada. Email:wbecker@ucalgary.ca
}

doi: http://dx.doi.org/10.4021/jmc1177w and receiving multiple doses, he continued to experience generalized convulsions and required sedation with propofol and ventilatory assistance. Continuous electroencephalographic (EEG) monitoring later revealed that his seizures had terminated after initiation of propofol and he remained seizure free post extubation.

His past medical history was significant for alcohol abuse with frequent prior admissions for alcohol withdrawal. There was a history of marijuana usage and heroin consumption. Additionally he was being managed for hepatic cirrhosis secondary to alcoholic liver disease and Hepatitis C infection. Clinic notes revealed that his hepatic disease had been stable with no radiologic evidence of malignancy.

The patient continued to be seizure free both clinically and through continuous EEG monitoring after his sedation was discontinued. As he continued to improve and his sedation wore off, he was noted to have significant cognitive deficits in terms of both retrograde and anterograde amnesia. His score on Montreal Cognitive Assessment was 14/30 with significant deficits in memory/delayed recall, visioconstruction skills and orientation. There was evidence of mild cerebellar dysfunction and a peripheral sensory neuropathy on clinical examination.

Admission laboratory investigations were significant for mildly elevated transaminase and an ammonia level of 104 $\mu \mathrm{mol} / \mathrm{L}$ (reference range 12 - $47 \mu \mathrm{mol} / \mathrm{L}$ ).Cerebrospinal fluid was acellular, protein and glucose were normal, and gram stain and Herpes Simplex Viral PCR were negative. His MRI findings are shown below.

He continued to be seizure free on maintenance phenytoin and later was switched to levetiracetam; however no improvement in his memory was noted. He had been treated with thiamine supplementation since presentation. Another EEG done at 1 month had revealed neither ongoing epileptiform activities nor any focal slowing. His metabolic parameters were within normal limits except for a persistently elevated ammonia level in the range of $99-108 \mu \mathrm{mol} / \mathrm{L}$.

However the patient continued to have persistent and profound memory impairment. His retrograde amnesia was dated approximately to be 20 years pre-event and he continued to have anterograde amnesia where his memory was lost after distraction. He could recall none of three objects after 


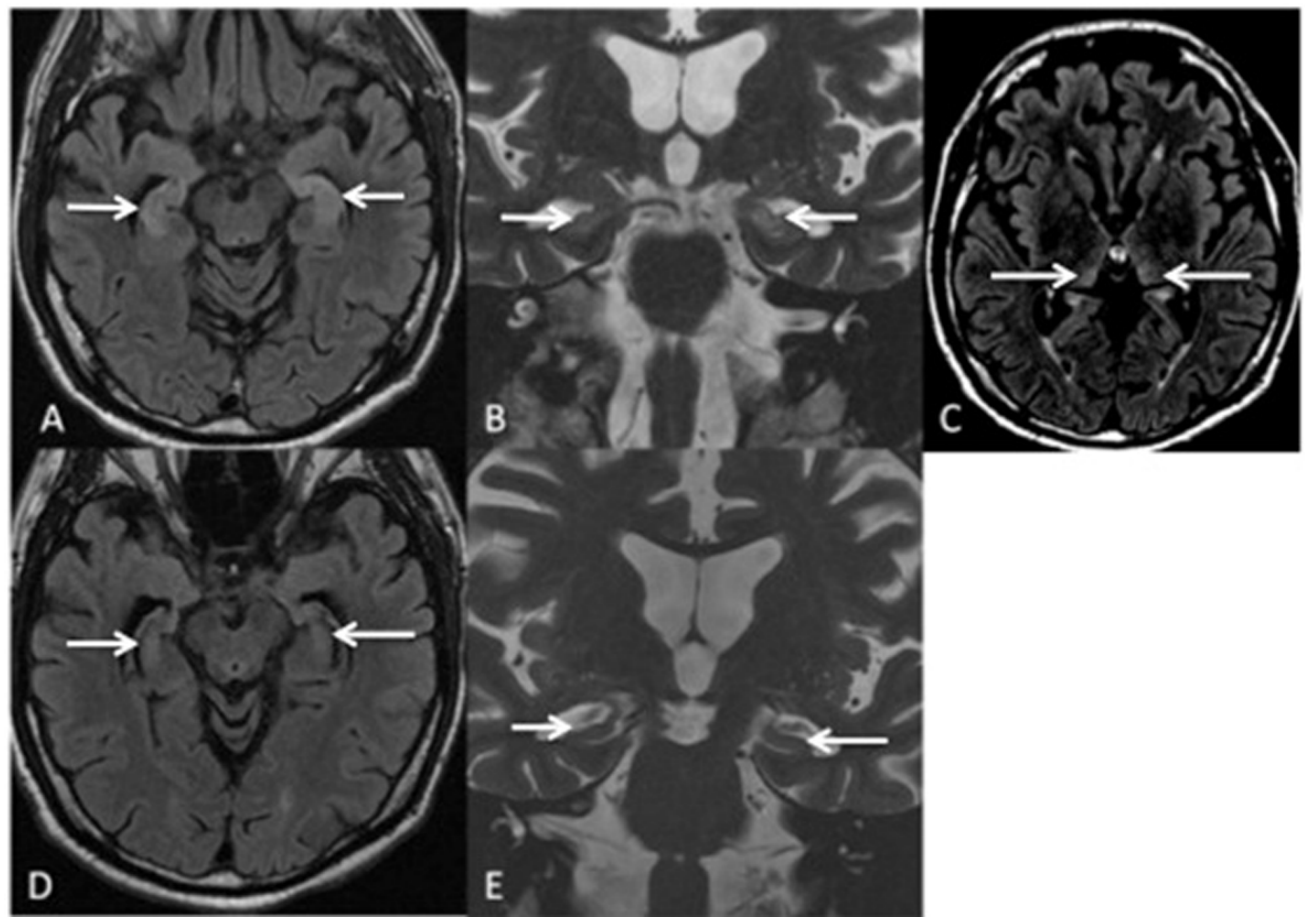

Figure 1. MRI Brain at 3 weeks post seizure episodes shows hyperintensity on the axial FLAIR (A) and coronal T2W imaging (B) in bilateral hippocampal heads as well as subtle hyperintensity in the posteromedial thalami bilaterally on the axial FLAIR imaging, (C). Follow up MRI shows hippocampal atrophy bilaterally on axial FLAIR (D), and coronal T2W imaging (E)

distraction.

His progressions of MRI findings are shown below (Fig. 1).

\section{Discussion}

The MRI findings correlate well with his persistent neurologic deficits of retrograde and anterograde amnesia. The role of the hippocampus in memory loss has been well established. Scoville et al in a case series of patients undergoing various degrees of medial temporal lobe resections showed that the degree of memory loss appeared to depend on the extent of hippocampal removal and that unilateral removal resulted in no lasting memory loss [1]. The patient highlighted above continued to have memory deficits with no improvement after status epilepticus and sequential MRIs revealed the transition from bilateral hippocampal inflammation to bilateral mesial temporal lobe sclerosis.

MRI changes in the paediatric population with febrile status epilepticus have been well described. Provenzale et al found that markedly hyperintense hippocampi in children with febrile status epilepticus were highly associated with mesial temporal lobe sclerosis [2]. A case report by Gong et al showed that progressive hippocampal atrophy can occur following a single event of status epilepticus in the adult population [3].

The sequence of MRI findings in our patient mirrored the observations made by the above mentioned case and correlated with his clinical picture. Alcohol and his risk of Korsakoff's syndrome are a potential confounding variable in this case presentation. Previous literature has always emphasized the role of various thalamic nuclei and other diencephalic structures, and undervalued the significance of the hippocampus in Korsakoff's syndrome. Sullivan et al measured hippocampal volumes in patients with Korsakoff's syndrome (KS), Alzheimer's Dementia and normal health controls and demonstrated that relative to control subjects the KS group had a -1 SD deficit in hippocampal volume bilaterally [4].

While the association between hippocampal atrophy and prolonged status epilepticus has already been described, this case report adds to the few isolated published cases that indicate that status epilepticus can cause hippocampal atrophy. No previous case report has demonstrated such a profound and persistent amnesia. The progression of his hippocampal inflammation and subsequent atrophy certainly cannot be explained by his alcoholism alone, although the findings of Sullivan et al would suggest that alcoholism and potentially thiamine deficiency may have contributed to both the clinical and radiological course of his disease. 


\section{Authors' Contributions}

The case presentation was not funded and there aren't any competing interests. Dr. Panday, Dr Becker nor Dr. Modi has any disclosures. 1) Dr. Panday was responsible for conception and design, drafting and revising the article as well as analysis of data. 2) Dr. Becker was responsible for drafting the article and final edit/approval. 3) Dr. Modi was responsible for analysis and interpretation of data.

\section{Competing Interests}

Dr. Werner J. Becker reports neither disclosures nor competing interests; Dr. Avidesh Panday reports neither disclosures nor competing interests; Dr. Modi reports neither disclosures nor competing interests.

\section{References}

1. Scoville WB, Milner B. Loss of recent memory after bilateral hippocampal lesions. 1957. J Neuropsychiatry Clin Neurosci. 2000;12(1):103-113.

2. Provenzale JM, Barboriak DP, VanLandingham K, MacFall J, Delong D, Lewis DV. Hippocampal MRI signal hyperintensity after febrile status epilepticus is predictive of subsequent mesial temporal sclerosis. AJR Am J Roentgenol. 2008;190(4):976-983.

3. Gong G, Shi F, Concha L, Beaulieu C, Gross DW. Insights into the sequence of structural consequences of convulsive status epilepticus: a longitudinal MRI study. Epilepsia. 2008;49(11):1941-1945.

4. Sullivan EV, Marsh L. Hippocampal volume deficits in alcoholic Korsakoff's syndrome. Neurology. 2003;61(12):1716-1719. 